\title{
Anchor Levels as a New Tool for the Theory and Measurement of Multiattribute Utility
}

\author{
Peter P. Wakker, Sylvia J. T. Jansen, Anne M. Stiggelbout \\ Medical Decision Making Department, Leiden University Medical Center, Leiden, The Netherlands \\ \{wakker@fee.uva.nl, s.jansen@otb.tudelft.nl, a.m.stiggelbout@lumc.nl\}
}

\begin{abstract}
This paper introduces anchor levels as a new tool for multiattribute utility theory. Anchor levels are attribute 1 levels whose values are not affected by other attributes. They allow for new interpretations and generalizations of known representations and utility measurement techniques. Generalizations of earlier techniques can be obtained because cases with complex interactions between attributes can now be handled. Anchor levels serve not only to enhance the generality, but also the tractability, of utility measurements, because stimuli can better be targeted toward the perception and real situation of clients. In an application, anchor levels were applied to the measurement of quality of life during radiotherapy treatment, where there are complex interactions with what happens before and after. Using anchor levels, the measurements could be related exactly to the situation of the clients, thus simplifying the clients' cognitive burden.
\end{abstract}

Key words: utility measurement; multiattribute utility; time preference; utility independence; QALY measurement

History: Received on August 20, 2003. Accepted by Don Kleinmuntz and Bob Clemen on September 13, 2004, after 3 revisions.

\section{Introduction}

This paper introduces anchor levels as a new tool for the theory and measurement of multiattribute utility. Anchor levels are special attribute levels whose utility is unaffected by interactions with other decision criteria (attributes). By imposing independence conditions only on the anchor levels and not on complete attributes, we generalize existing approaches.

Anchor levels give new interpretations and generalizations of existing results, such as Fishburn's (1965) additive decomposability and Anscombe and Aumann's (1963) subjective expected-utility model. By means of anchor levels, we can generalize existing measurement techniques. We can define and measure attribute utilities when the attributes have interactions of high complexity.

Peter P. Wakker is currently affiliated with the Department of Economics, University of Amsterdam, Roestersstraat 11, Amsterdam, 1018 WB, The Netherlands.

Sylvia J. T. Jansen is currently affiliated with the Research Institute OTB, Methodology and Informatics, Delft, The Netherlands.
The primary application of anchor levels to utility measurement concerns situations where the utility of some attribute, rather than an aggregate overall utility, is the primary topic of interest, for instance, because this attribute utility is the quantity relevant to other decisions. Jansen et al. $(1998,2000)$ informally applied our technique to intertemporal preferences in a study of the stability over time of quality of life during radiotherapy treatment. This application is described in greater detail later in this paper. For intertemporal choice, Kahneman (1994) emphasized the importance of measuring instant utility-i.e., the experienced well-being at given time points-as opposed to the overall utility of entire episodes. Another example arises in decision under uncertainty. For standard utility-measurement techniques such as the standard gamble, our primary interest concerns the utility of the outcomes, and not the expected utilities of the gambles used during the measurement procedure. Those gambles serve only as a tool to measure the utilities of the outcomes.

In Jansen et al. (1998, 2000), anchor levels not only allowed for utility measurement in the presence of interactions, but also permitted construction of stim- 
uli familiar to the subjects. The present paper is a formalization of this application, with the aim to demonstrate that these techniques are of use for general multiattribute utility theory. Other applications will be mentioned, such as the measurement of utility when expected utility's separability is violated in decision under uncertainty, the measurement of individual happiness when this happiness depends on the happiness of other individuals in society, and the identifiability of probabilities under state-dependent expected utility.

Interactions between attributes have been studied extensively in multiattribute utility theory. Keeney and Raiffa (1976) presented multilinear models with multiplicative interactions. These models were used in a number of empirical studies (Fryback and Keeney 1983, Torrance et al. 1996). The multivalent and hypercube models developed by Farquhar and Fishburn (1981) allow for more general interactions. These general interactions, however, complicate the measurement of utility.

For applications to utility measurement, the message of this paper can be stated concisely: Determine levels of attributes that are affected by interactions as little as possible, and then use Equations (4.3)-(4.5) in $\S 4$ to measure utility. The rest of this paper provides examples, preference axioms, and a quantitative representation to support this method. Apart from the assumed presence, or constructibility, of anchor levels, no restrictions are imposed on the other attribute levels, and every kind of general interaction is permitted.

The outline of this paper is as follows. Section 2 describes separability and attribute interaction in multiattribute utility theory. Section 3 demonstrates how the utility contribution of anchor levels can be separated additively, without requiring additive separability for the utility contribution of other attribute levels. Additive decomposability holds if and only if all attribute levels are anchor levels, a condition that is somewhat weaker than Fishburn's (1965) marginal independence. Section 4 formally presents our new method, which assumes that only some levels are anchor levels. It establishes the measurability of attribute utilities through a preference foundation. Section 5 considers attribute-independent anchor levels, i.e., anchor levels that can be used across different attributes. We generalize Anscombe's and
Aumann's (1963) preference axiomatization of subjective expected utility for decision under uncertainty (events with unknown probabilities). As these authors did, we assume the von Neumann-Morgenstern axioms for known probabilities, implying expected utility there. We then show that subjective expected utility also holds for events with unknown probabilities if and only if all attribute levels ("outcomes") are attribute-independent anchor levels. This provides a new preference foundation for Bayesian decision analysis.

Mathematical examples are provided throughout to illustrate the basic meaning of concepts presented in this paper. In Example 4.4, the importance weights of the attributes have a natural interpretation as discounted duration and the interaction-dependent utility functions as quality of life. Example 4.5 illustrates the notorious problem of nonidentifiability of probability for state-dependent expected utility. Indeed, the parameters of our model are theoretical scaling factors without clear interpretation in this example. Example 5.4 illustrates the general concept of attribute-independent anchor levels. In this example, the importance weights and utilities are regular subjective probabilities and utilities in the subjective expected-utility model. Example 5.6 considers a violation of separability due to disappointment. Classical expected utility is violated and its elicitation techniques cannot be used, but the techniques presented here can still be used to measure probabilities and utilities, including the disappointment effect. The psychological interpretation of such interactions is not revealed by the preference models, but should be based on context and application.

Following a short history of decision analysis in the health domain in $\S 6, \S 7$ shows how anchor levels can resolve problems in the measurement of quality-of-life years (QALYs) in the health domain. The section formalizes the approach of Jansen et al. (2000). Section 8 presents a discussion, and $\$ 9$ concludes. Proofs are in the appendix.

\section{Interactions in Multiattribute Utility}

Multiattribute utility theory provides tools for aggregating different objectives that may be mutually competitive into an overall decision (Keeney and Raiffa 
1976, von Winterfeldt and Edwards 1986). Tradeoffs usually have to be made between the several objectives, or attributes as they will be called formally. A larger house may be farther away from work, a better career opportunity may offer a lower job security, etc. Formally, we assume $n$ attributes and consider a set $X=X_{1} \times \cdots \times X_{n}$ of alternatives. Alternatives are $n$-tuples $\left(x_{1}, \ldots, x_{n}\right)$ where $x_{j}$ designates the level of the alternative on attribute $j$. For example, there may be $n$ time periods, with $x_{j}$ designating the health state during period $j$.

In most applications, alternatives are evaluated additively (Keeney and Raiffa 1976, Equation (6.29); Stigler 1950, §§IV and VIII B; von Winterfeldt and Edwards 1986, Equation (8.1)). That is,

$$
U\left(x_{1}, \ldots, x_{n}\right)=\sum_{j=1}^{n} w_{j} u_{j}\left(x_{j}\right)
$$

evaluates the alternative $\left(x_{1}, \ldots, x_{n}\right)$, where $U\left(x_{1}\right.$, $\left.\ldots, x_{n}\right)$ is the overall utility of the alternative, $u_{j}\left(x_{j}\right)$ is the utility of $x_{j}$, and $w_{j}$ is a weighting factor to settle the exchange rates between the various attributes. In the QALY (quality-of-life years) model that is commonly used in medical cost-effectiveness studies, $u_{j}\left(x_{j}\right)$ is the quality of life when being in health state $x_{j}$ and $w_{j}$ corresponds to the discounted duration of period $j$. The Markov models commonly used to evaluate stochastic processes essentially require additive separability over disjoint periods (Johannesson et al. 1996, p. 286) as in Equation (2.1).

The additive model in Equation (2.1) holds only if appropriate preference conditions, such as utility independence and separability, are satisfied. The latter condition concerns riskless options and entails, for instance, that with $\succcurlyeq$ denoting preference,

$$
\begin{aligned}
& \left(c_{1}, x_{2}, \ldots, x_{n}\right) \succcurlyeq\left(c_{1}, y_{2}, \ldots, y_{n}\right) \\
& \quad \text { if and only if }\left(c_{1}^{\prime}, x_{2}, \ldots, x_{n}\right) \succcurlyeq\left(c_{1}^{\prime}, y_{2}, \ldots, y_{n}\right) .
\end{aligned}
$$

That is, preference is independent of the common level $\left(c_{1}\right.$ or $\left.c_{1}^{\prime}\right)$ of the first attribute. Utility independence is a similar condition, but refers to risky preferences. These conditions exclude interactions between attributes. The following mathematical example and its extensions in later sections will illustrate the concepts of this paper.
ExAmple 2.1. Assume $n=3, X_{1}=X_{2}=X_{3}=[0,1]$, and expected utility with $U\left(x_{1}, x_{2}, x_{3}\right)=x_{1}+x_{2}+$ $x_{3}+1-\exp \left(-x_{1} x_{2} x_{3}\right)$. The last term generates interactions between the attributes. We have $(1,0,1) \prec$ $(1,0.45,0.45)$ and $(0,0,1) \succ(0,0.45,0.45)$, so that preference is not independent of the common level of the first attribute. This violation of Equation (2.2) shows that $U$ is not additively decomposable, and neither is a strictly increasing transform of an additively decomposable function.

If additivity is violated, then it is sometimes reestablished by redefining the attributes (Keeney 1992, McDaniels 1995, p. 421). In applications, deviations from additivity can be ignored as long as they do not generate large biases (Dolan 2000, pp. 1754-1755). In many cases, however, the biases are too large to be ignored. Such cases are common, for instance, in time preferences (Loewenstein and Elster 1992, Prelec and Loewenstein 1991). Many phenomena in economics, such as addiction and habit formation, are driven by the nonseparability of disjoint periods (Becker 1996).

In the health domain, the utility measurement of temporary health states is similarly complex because this utility often depends on what happens before and after, a dependence that directly violates separability (Dolan 2000, p. 1743; Krabbe and Bonsel 1998; Kupperman et al. 1997; Loomes and McKenzie 1989, p. 303; Richardson et al. 1996). Broome (1993, pp. 151152) considers separability to be "the most dubious condition" of the QALY model. Many violations of sophisticated requirements of the classical QALY model also imply a violation of the more basic separability. Examples are the dependency of quality of life on: (a) prognosis (Torrance 1987, p. 596; Krabbe and Bonsel 1998, pp. 182-183); (b) prior experience with the health state (Dolan 2000, §3.2.3; Gold et al. 1996, p. 100; Sackett and Torrance 1978, p. 703; Stiggelbout and de Haes 2001, pp. 226-227); (c) the duration spent in this health state (Dolan and Gudex 1995; McNeil et al. 1981; Miyamoto and Eraker 1988, p. 15; Sackett and Torrance 1978, p. 703; Sutherland et al. 1982).

A general equation for expressing interactions between attributes is

$$
U\left(x_{1}, \ldots, x_{n}\right)=\sum_{j=1}^{n} w_{j} u_{j}\left(x_{j}, x\right),
$$


where $u_{j}\left(x_{j}, x\right)$ is the utility of $x_{j}$, which depends on the levels of the other attributes $x_{1}, \ldots, x_{j-1}$, $x_{j+1}, \ldots, x_{n}$. Without further specifications, Equation (2.3) is too general to yield empirical predictions. It is impossible to elicit the weights $w_{j}$ or the utilities $u_{j}\left(x_{j}, x\right)$ from decisions. Only the total sum $\sum_{j=1}^{n} w_{j} u_{j}\left(x_{j}, x\right)$ can be elicited. Further specifications that do allow for the elicitation of the parameters $w_{j}$ and $u_{j}\left(x_{j}, x\right)$ are the topic of this paper.

In some situations, separability and utility independence cannot be maintained in full strength, but weakened versions can be maintained. In such cases it is commonly assumed in the literature that some attributes are separable from (so have utilities independent of) some other attributes, but interactions between remaining attributes are permitted (Leontief 1947, Strotz 1957). Keeney and Raiffa (1976) extensively studied similar weakenings of utility independence. Interactions were multiplicative in their models and were governed by a restricted number of extra parameters.

What the mentioned weakenings do have in common is that those parts of separability or utility independence that are maintained are invariably imposed on all levels of the attributes in question. For example, if attributes $2, \ldots, n$ are separable from attribute 1-i.e., Equation (2.2) holds-then the requirement should hold for all levels $c_{1}, c_{1}^{\prime}$, and for all $x_{2}, \ldots, x_{n}$, $y_{2}, \ldots, y_{n}$. Such conditions are not satisfied in Example 2.1. In this respect, our paper will introduce a new approach. We introduce a version of separability that is imposed only on specially chosen levels of attributes, the anchor levels, and not on other levels. Then, a meaningful measurement of attribute utility $u_{j}\left(x_{j}, x\right)$ remains possible even if this utility depends on the other levels $x_{1}, \ldots, x_{j-1}, x_{j+1}, \ldots, x_{n}$. In applications, the anchor levels should be chosen with care so as to be suited for this purpose.

\section{Partially Additive Decomposability Through Anchor Levels}

This paper follows the Keeney and Raiffa (1976) approach to multiattribute utility, where risk is assumed to be present in the decision process. We therefore consider not only the set $X_{j=1}^{n} X_{j}$ of alternatives-i.e., combinations of conceivable attribute levels-but, more generally, the set $L\left(X_{j=1}^{n} X_{j}\right)$ of all prospects over alternatives. Prospects are simple probability distributions over $X_{j=1}^{n} X_{j}$, where simple means that the number of possible alternatives is finite. A typical prospect is $\left(p_{1}: x^{1} ; \ldots ; p_{m}: x^{m}\right)$, yielding alternative $x^{j}=\left(x_{1}^{i}, \ldots, x_{n}^{j}\right)$ with probability $p_{j}$, for $j=1, \ldots, m$. Probabilities $p_{1}, \ldots, p_{m}$ are nonnegative and sum to one. By $\succcurlyeq$ we denote the preference relation of an agent over the prospects; strict preference $\succ$ and equivalence/indifference $\sim$ are as usual. Expected utility means that there exists a utility function $U: X_{i=1}^{n} X_{i} \rightarrow \mathbb{R}$ on the alternatives such that preferences maximize

$$
\left(p_{1}: x^{1} ; \ldots ; p_{m}: x^{m}\right) \mapsto \sum_{j=1}^{m} p_{j} U\left(x^{j}\right) .
$$

We assume Equation (3.1) throughout this paper. That is, we assume expected utility, as is commonly done in classical multiattribute utility theory and in most normative works. Extensions of multiattribute utility to nonexpected-utility models include Bleichrodt and Miyamoto (2003), Dyckerhoff (1994), Fishburn and LaValle (1992), and Zank (2001). Similar extensions of our results are left to future studies. This paper focuses on the decomposition of the overall utility function $U\left(x_{1}, \ldots, x_{n}\right)$ into attribute utility functions $u_{j}\left(x_{j}\right)$, which is the general topic of multiattribute utility.

The most well-known preference condition for the characterization of additive decomposability is Fishburn's (1965) marginal independence (see also Keeney and Raiffa 1976, Theorem 6.4). It requires that the utility of a prospect $\left(p_{1}: x^{1} ; \ldots ; p_{m}: x^{m}\right)$ depend only on the marginal distributions $\left(p_{1}: x_{i}^{1} ; \ldots ; p_{m}: x_{i}^{m}\right)$ generated over the attributes $i=1, \ldots, n$, and not on the correlations or joint distributions of the attributes otherwise. For the purpose of this paper, a weakened condition based upon Fishburn's (1965) Theorems 1 and 3 is most suited. We will discuss the condition in more detail, and we first introduce a preparatory notation. The notation deviates from the conventions of Keeney and Raiffa (1976), but is convenient and is commonly used today. For $x=\left(x_{1}, \ldots, x_{n}\right)$ and $y_{i} \in X_{i}, y_{i} x$ is $x$ with $x_{i}$ replaced by $y_{i}$; i.e., it is $\left(x_{1}, \ldots, x_{i-1}, y_{i}, x_{i+1}, \ldots, x_{n}\right)$.

Consider the $50-50$ prospect $\left(1 / 2: b_{i} x ; 1 / 2: b_{i} y\right)$. The two alternatives have a common $i$ th attribute $b_{i}$. Imagine that an agent can choose whether the left or the right attribute level $b_{i}$ is improved into another, 
better, level $g_{i}$. It seems intuitively plausible that, if there is no interaction with the other attributes, then the utility improvement is the same in the alternative with $x \mathrm{~s}$ as with $y \mathrm{~s}$. That is, the following indifference (denoted by $\sim$ ) seems to be indicative of the absence of interaction.

$$
\left(1 / 2: g_{i} x ; 1 / 2: b_{i} y\right) \sim\left(1 / 2: b_{i} x ; 1 / 2: g_{i} y\right)
$$

We call $b_{i}, g_{i}$ (a pair of) anchor levels (for attribute $i$ ) if Equation (3.2) is satisfied for all $x, y$. They are nontrivial if not $g_{i} x \sim b_{i} x$ for some $x$ (which, hence, holds for all $x$ ).

Substituting expected utility shows that, equivalently,

$$
U\left(g_{i} x\right)-U\left(b_{i} x\right) \text { is independent of } x .
$$

Equation (3.3) suggests that the replacement of $b_{i}$ by $g_{i}$ can serve as a standard for the utility unit, invariant in all contexts. Equation (3.2) provides an empirical test. Nontriviality implies that the utility difference in Equation (3.3) is not zero.

ExAMPLE 3.1. Assume Example 2.1, but now with prospects added and expected utility with respect to the function $U$ as defined in Example 2.1. Preferences for prospects over outcomes $\left(c, 1, x_{3}\right)$ are risk neutral in $x_{3}$ for $c=0$, but strictly risk averse for $c>0$. This constitutes a violation of the weakest utility independence conditions in Keeney and Raiffa (1976), and illustrates once more the interaction between attributes. For the prospect $(1 / 2:(0,1,1) ; 1 / 2:(0,1,0))$, with $i=1, b_{1}^{\prime}=0$, and $g_{1}^{\prime}=1$, the improvement of the first attribute of the left alternative is preferred to that of the right alternative; i.e.,

$$
\begin{aligned}
(1 / 2: & (\mathbf{1}, 1,1) ; 1 / 2:(0,1,0)) \\
& \quad(1 / 2:(0,1,1) ; 1 / 2:(\mathbf{1}, 1,0)),
\end{aligned}
$$

violating Equation (3.2). Hence, $b_{1}^{\prime}$ and $g_{1}^{\prime}$ are not anchor levels. It can be seen that there exist no nontrivial anchor levels in this example.

Violations of Equation (3.2), like those in the above example, have been used to define multivariate risk aversion. For instance, Richard (1975, conditions (i) and (ii) on p. 13) considered the case of two attributes. Multivariate risk aversion was defined as a preference for the side in Equation (3.2) where, for $j \neq i$, the preferred level of attribute $j(x$ or $y$ ) is coupled with the nonpreferred level of attribute $i$ ( $g$ or $b$ ). Such a condition had been considered before by de Finetti (1932) and has been tested in several studies (Payne et al. 1984; Pliskin et al. 1980, p. 210).

In Example 3.1, no nontrivial anchor levels existed. The following example shows that it is possible, at least in theory, to add auxiliary attribute levels that can serve as anchor levels.

Example 3.2. Assume Example 3.1. We extend the set $X_{1}$ by adding the attribute level $g_{1}=2$, so that the attribute set $[0,1] \cup\{2\}$ results. Assume that

$$
U\left(2, x_{2}, x_{3}\right)=4+U\left(0, x_{2}, x_{3}\right){ }^{1}
$$

With $b_{1}=0,\left\{b_{1}, g_{1}\right\}$ are anchor levels for the first attribute, as follows from Equation (3.3).

A set of attribute levels are anchor levels if each pair from the set is a pair of anchor levels. The following theorem shows that anchor levels do not interact with other attributes, and are additively separable.

Theorem 3.3. Let $1 \leq i \leq n . A_{i}$ is a set of anchor levels for attribute $i$ if and only if there exist functions $V$ : $\prod_{j \neq i} X_{j} \rightarrow \mathbb{R}$ and $v_{i}: A_{i} \rightarrow \mathbb{R}$, such that $U(x)=v_{i}\left(x_{i}\right)+$ $V\left(x_{1}, \ldots, x_{i-1}, x_{i+1}, \ldots, x_{n}\right)$ whenever $x_{i} \in A_{i}$.

In Example 3.2,

$$
\begin{aligned}
& i=1, \quad A_{1}=\{0,2\}, \quad V\left(x_{2}, x_{3}\right)=x_{2}+x_{3}, \quad v_{1}(0)=0, \\
& \text { and } v_{1}(2)=4 .
\end{aligned}
$$

Corollary 3.4.

(i) $U(x)$ can be written as $v_{i}\left(x_{i}\right)+V\left(x_{1}, \ldots, x_{i-1}\right.$, $\left.x_{i+1}, \ldots, x_{n}\right)$ for some functions $v_{i}$ and $V$, if and only if all attribute levels $x_{i} \in X_{i}$ are anchor levels.

(ii) The additive decomposition (Equation (2.1)) holds if and only if all levels of all attributes are anchor levels.

Statement (ii) is a variation of Fishburn's (1965, Theorem 3) characterization of additive decomposability. The condition in the statement is obviously weaker than Fishburn's marginal independence. The latter condition immediately implies our conditioni.e., Equation (3.2) — for all attribute levels, because all

\footnotetext{
${ }^{1}$ We chose the addition of 4 in this example so as to ensure monotonicity in all attributes. Calculations show that, even stronger, $U(2,0,0)>U(1,1,1)$, so that $U\left(2, x_{2}, x_{3}\right)>U\left(y_{1}, y_{2}, y_{3}\right)$ whenever $y_{1}<2$.
} 
marginal distributions are the same in Equation (3.2). Our condition is weaker because it only considers the special case of 50-50 two-outcome marginal distributions with identical joint distributions off attribute $i$. Our condition still implies additive decomposability and, thus, marginal independence.

In a number of papers, Farquhar and Fishburn derived representations from generalizations-i.e., lessrestrictive versions, of the above conditions (see Farquhar and Fishburn 1981, and the references therein). In their multivalent representations, attribute sets $X_{i}$ are partitioned into subsets $X_{j}^{i}$ such that Equation (3.3) holds for all elements $b_{i}, g_{i}$ from each subset $X_{j}^{i}$. Then, generalized (multivalent) additive representations can be derived on subdomains $X_{1}^{i_{1}} \times \cdots \times X_{n}^{i_{n}}$. The fractional hypercube methods used generalized forms of Equation (3.2), with multioutcome prospects. Various utility functions, constructed through several additions and multiplications, were derived. For a more general form than in Corollary 3.4(i), see Theorem 3 of Farquhar and Fishburn (1981).

Although anchor levels themselves are additively separable, their corresponding attribute need not be, and any kind of interaction is conceivable for the other levels of this attribute. In Jansen et al. (1998, 2000), a health state "hospitalization caused by a serious accident" was not present in the original domain of their study, but was added to serve as an anchor level, in the same way that attribute level $g$ was added in Example 3.2. Further details are in $§ 7$.

\section{Using Anchor Levels to Measure Utility}

In the preceding section, we saw that all attribute levels must be anchor levels under additive decomposability. The multivalent and hypercube methods of Farquhar and Fishburn (1981) extended this result to partitions of attribute levels and more complex representations. In this section, we will follow an alternative route from Equation (3.3).

We assume for now that $\left\{b_{i}, g_{i}\right\}$ are nontrivial anchor levels with $g_{i} x>b_{i} x$. Here $g$ abbreviates good and $b$ abbreviates bad. We pursue a definition of the utility of attribute $x_{i}$ within $x$, even though there are interactions. We keep all attributes $j \neq i$ fixed at their level $x_{j}$, and define $u_{i}\left(x_{i}, x\right)$ to be $U\left(x_{i} x\right)$, but renormalized to be zero at $b_{i} x$ and one at $g_{i} x$. In other words,

$$
u_{i}\left(x_{i}, x\right)=\frac{U(x)-U\left(b_{i} x\right)}{U\left(g_{i} x\right)-U\left(b_{i} x\right)} .
$$

Defining $V\left(x_{1}, \ldots, x_{i-1}, x_{i+1}, \ldots, x_{n}\right)=U\left(b_{i} x\right)$ and $w_{i}=U\left(g_{i} x\right)-U\left(b_{i} x\right)$, we get

$U(x)=w_{i} u_{i}\left(x_{i}, x\right)+V\left(x_{1}, \ldots, x_{i-1}, x_{i+1}, \ldots, x_{n}\right)$.

Not only is the location term $V\left(x_{1}, \ldots, x_{i-1}, x_{i+1}\right.$, $\left.\ldots, x_{n}\right)$ independent of $x_{i}$, but also, by Equation (3.3), the scaling factor $w_{i}$ is independent of $x ; w_{i}$ depends only on $i$, given $g_{i}$ and $b_{i}$. The factor $w_{i}$ can be interpreted as the weight of attribute $i$. The linear decomposition is genuine in the sense that all interactions are captured through $u_{i}\left(x_{i}, x\right)$, the attribute utility of $x_{i}$ at $x$ (relative to $b$ and $g$ ).

Given expected utility, we can measure $u_{i}\left(x_{i}, x\right)$ as follows, always with the probability $0 \leq p \leq 1$ such that the relevant indifference results.

Case 1.

$$
\begin{aligned}
& U(x) \geq U\left(g_{i} x\right) \text { and } \\
& \quad g_{i} x \sim\left(p: x ; 1-p: b_{i} x\right): u_{i}\left(x_{i}, x\right)=\frac{1}{p} \geq 1 .
\end{aligned}
$$

Case 2.

$$
\begin{aligned}
& U\left(g_{i} x\right) \geq U(x) \geq U\left(b_{i} x\right) \text { and } \\
& \quad x \sim\left(p: g_{i} x ; 1-p: b_{i} x\right): 0 \leq u_{i}\left(x_{i}, x\right)=p \leq 1 .
\end{aligned}
$$

Case 3.

$$
\begin{aligned}
U\left(b_{i} x\right) & \geq U(x) \text { and } \\
b_{i} x & \sim\left(p: g_{i} x ; 1-p: x\right): u_{i}\left(x_{i}, x\right)=\frac{-p}{1-p} \leq 0 .
\end{aligned}
$$

Example 4.1. Assume Example 3.2. Imagine that we want to measure the utility of the first attribute levels 0, 0.5, and 1. Classical measurement methods are based on utility independence, and would use an equivalence such as

$$
(0.5,1,0) \sim(0.500:(1,1,0) ; 0.500:(0,1,0))
$$

to conclude that $0.5^{\prime}$ s utility is the midpoint of the utilities of 0 and 1 , suggesting linearity and risk neutrality. However, if we change the third attribute level to 1 , then we obtain an equivalence

$$
(0.5,1,1) \sim(0.547:(1,1,1) ; 0.453:(0,1,1)),
$$


suggesting that 0.5 's utility now exceeds the midpoint of the utilities of 0 and 1 and, thus, suggesting concavity and risk aversion. The deviation from Equation (4.6) is considerable. A traditional measurement through Equation (4.6), based on utility independence, would lead to wrong conclusions if $x_{2}=$ $x_{3}=1$ in the relevant context of application.

If we use the anchor levels defined before, $b_{1}=0$ and $g_{1}=2$, then we find, in general,

$$
\begin{gathered}
w_{1}=4, \quad V\left(x_{2}, \ldots, x_{n}\right)=U\left(0, x_{2}, \ldots, x_{n}\right), \\
u_{1}\left(b_{1}, x\right)=0, \quad \text { and } u_{1}\left(g_{1}, x\right)=1
\end{gathered}
$$

as usual, and

$$
u_{1}\left(x_{1}, x\right)=\left(U\left(x_{1}, x_{2}, \ldots, x_{n}\right)-U\left(0, x_{2}, \ldots, x_{n}\right)\right) / 4 .
$$

The interactions between the attributes have now been incorporated explicitly. For example, at $x=$ $(1,1,1)$, we obtain

$$
\begin{aligned}
u_{1}(0, x) & =0, \\
u_{1}(0.5, x) & =0.223, \\
u_{1}(1, x) & =0.408,
\end{aligned}
$$

and

$$
\frac{u_{1}(0.5, x)}{u_{1}(1, x)-u_{1}(0, x)}=0.547,
$$

in agreement with the indifference in Equation (4.7). At $x$, the utility of the first attribute level 0.5 clearly exceeds the midpoint of the utilities of 1 and 0 , and $u_{1}(\cdot, x)$ is concave.

Throughout the rest of this paper, $u_{j}\left(y_{j}, x\right)$ designates the (attribute) utility of $y_{j}$ when the levels of the other attributes are $x_{1}, \ldots, x_{j-1}, x_{j+1}, \ldots, x_{n}$, in keeping with the preceding analysis. Obviously, $x_{j}$ plays no role in this notation. The following theorem establishes the empirical meaningfulness of the preceding constructions. It formalizes what was illustrated in Example 4.1.

Theorem 4.2. Assume that $g_{i} r \succ b_{i} r$ for some $r$. Then $\left\{g_{i}, b_{i}\right\}$ are anchor levels if and only if

$$
U(x)=w_{i} u_{i}\left(x_{i}, x\right)+V\left(x_{1}, \ldots, x_{i-1}, x_{i+1}, \ldots, x_{n}\right),
$$

where:

(i) $w_{i}>0$ is independent of $x$.

(ii) $u_{i}\left(x_{i}, x\right)$ is given by Equations (4.3)-(4.5). (iii) $U\left(y_{i} x\right)-U\left(z_{i} x\right)=w_{i}\left(u_{i}\left(y_{i}, x\right)-u_{i}\left(z_{i}, x\right)\right)$ for all $y_{i}, z_{i}$.

Further, $u_{i}$ is uniquely determined. The uniqueness of $U$ up to a unit and location parameter corresponds to uniqueness of $w_{i}$ and $V$ up to the same unit and of $V$ up to the same location.

The theorem gives a kind of first-order Taylor expansion of $U$, be it that it is global and exact. $u_{i}\left(x_{i}, x\right)$ is the renormalized one-dimensional cut of the $n$ variable function $U$. It captures the marginal utility contribution of attribute $i$, incorporating the interactions with the other levels $x_{j}$. The factor $w_{i}$ restores the scale, and the function $V$ the location. $V$ is independent of $x_{i}$. Because $b_{i}$ and $g_{i}$ are anchor levels, $w_{i}$, the weight of attribute $i$, is independent of $x$ and $x_{i}$.

The following theorem shows that the above result can be obtained in an overall manner when anchor levels are available on all attributes. It thereby provides a special case of Equation (2.3) with all parameters empirically meaningful, while preserving the generality of Equation (2.3) apart from the restrictions for the anchor levels.

THeorem 4.3. Let $g, b$ be two alternatives with $g_{i} b \succ b$ for each $i$. Then all $\left\{b_{i}, g_{i}\right\}$ are anchor levels if and only if

$$
U\left(x_{1}, \ldots, x_{n}\right)=\sum_{j=1}^{n} w_{j} u_{j}\left(x_{j}, x\right)+W(x)
$$

where:

(i) The $w_{j}$ s are positive and independent of $x$;

(ii) $u_{i}\left(x_{i}, x\right)$ is given by Equations (4.3)-(4.5);

(iii) For each i, $U\left(y_{i} x\right)-U\left(z_{i} x\right)=w_{i}\left(u_{i}\left(y_{i}, x\right)-\right.$ $\left.u_{i}\left(z_{i}, x\right)\right)$ for all $y_{i}, z_{i}$. Equivalently,

$$
\sum_{j \neq i} w_{j} u_{j}\left(x_{j}, x\right)+W(x)
$$

is independent of $x_{i}$.

Further, the $u_{i}$ s are uniquely determined. The uniqueness of $U$ up to a unit and location parameter corresponds with uniqueness of the $w_{i}$ s and $W$ up to the same unit and of $W$ up to the same location.

The theorem again gives a kind of first-order Taylor expansion, with the $u_{j}\left(x_{j}, x\right)$ s normalized onedimensional cuts, the $w_{j}$ s scale factors independent of $x$, and $W$ jointly organizing the location terms for all attributes. For each $i, \sum_{j \neq i} w_{j} u_{j}\left(x_{j}, x\right)+W(x)$, which is 
independent of $x_{i}$, restores the location of $U$, and is the analog of $V$ in Theorem 4.2. The two statements in (iii) both capture the same idea-namely, that variation in the $i$ th attribute is completely captured through $w_{i}$ and $u_{i}$.

In decision models, the meaning of the parameters inferred from decisions is based on interpretations and extraneous information, and is always open to debate. In Savage's (1954) famous derivation of subjective expected utility, for instance, subjective probabilities are usually interpreted as degrees of belief and utilities as cardinal indexes of value. Discussions of the probability interpretation will be referenced in Example 4.5. Discussions of the utility interpretation include Dyer and Sarin (1982), Ellsberg (1954), Ng (1999), and many others. In our model, the extent to which $w_{i}$ is only a convenient scaling factor or is a factor with a special interpretation depends on the extent to which $U\left(g_{i} x\right)$ and $U\left(b_{i} x\right)$ have a special interpretation or not. In isolation, our decision model cannot speak to these questions, but extraneous information and interpretations in the context of application should decide. We give two examples to illustrate this point, one where $w_{i}$ has a natural interpretation, and one where it does not.

Example 4.4 (Time Preference for Health States). Assume that attributes designate periods, attribute levels designate health states during the corresponding periods, $g$ designates good health, and $b$ designates death or something with the same instant utility as being dead (e.g., being numb). It is a convention in the health domain that good health be assigned utility one, and death utility zero (Gold et al. 1996, pp. 84, 88). This convention is essential for obtaining meaningful comparisons of the effects of different medical treatments for different patient groups in cost-effectiveness studies. Torrance (1986, p. 17) wrote:

The central basis for this method is that the difference in utility between being dead and being healthy is set equal across people. In this way the method is egalitarian within the health domain; that is, each individual's health is counted equally.

Based on this convention, and in agreement with the normalization in Equations (4.3)-(4.5), we set $u_{i}\left(g_{i} x\right)=1$ and $u_{i}\left(b_{i} x\right)=0$.
The convention just described implicitly assumes that $b$ and $g$ are anchor levels. This convention can be justified only in cases where the preference condition in Equation (3.2) can be justified, which is a nontrivial requirement. In such cases, $w_{i}$ reflects the discounted duration of the corresponding period, and $u_{i}\left(x_{i}, x\right)$ can be interpreted as a quality-of-life indexi.e., a rate of utility per time unit when being in health state $x_{i}$ during period $i$, assuming health state $x_{j}$ for period $j, j \neq i$.

EXAMPLE 4.5 (Decision Under UnCERTAINTY WITH State-Dependent Expected Utility). There are $n$ states (of nature), of which exactly one is true, the others are not true, and the agent is uncertain about which is the true one. For example, each state of nature can describe a disease that a patient possibly has, given her symptoms. $X_{1}=\cdots=X_{n}=\mathscr{C}$ is an outcome set concerning the potential outcomes of medical treatments of the patient. Alternatives are also called acts. Act $\left(x_{1}, \ldots, x_{n}\right) \in X_{1} \times \cdots \times X_{n}=\mathscr{C}^{n}$ yields outcome $x_{j}$ if state of nature $j$ is true.

We assume a two-stage Anscombe-Aumann (1963) model, with $\left(p_{1}: x^{1} ; \ldots ; p_{m}: x^{m}\right)$ yielding act $x^{j}$ with probability $p_{j}$, for each $j$. For example, $\left(p_{1}: x^{1} ; \ldots\right.$; $\left.p_{m}: x^{m}\right)$ describes a medical treatment that with probability $p_{j}$ has effects as described by $x^{j}$. Then the $p_{j} \mathrm{~s}$ represent uncertainties that can be probabilized on the basis of extensive epidemiological data, and the states of nature represent uncertainties with unknown probabilities. As throughout this paper, $\left(p_{1}: x^{1} ; \ldots ; p_{m}: x^{m}\right)$ is evaluated by $\sum_{j=1}^{n} p_{j} U\left(x^{j}\right)$. Anscome and Aumann (1963) assumed probabilistic mixtures both before and after the states. Their Assumption 2 states that it is immaterial whether a mixture is modeled before or after the states.

We assume that $U\left(x_{1}, \ldots, x_{n}\right)=\sum_{j=1}^{n} r_{j} v_{j}\left(x_{j}\right)$ is a subjective expected utility with subjective probabilities $r_{1}, \ldots, r_{n}$ and state-dependent utility functions $v_{j}$. This state-dependent variation of Anscombe and Aumann's model was described by Arrow (1951, pp. 431-432) and axiomatized by Fishburn (1965), Rubin (1987), and Karni (1993).

Additive separability is satisfied, and all outcomes are anchor levels for all attributes ( $=$ states of nature). For each state $i$ we can define $w_{i}=r_{i}\left(v_{i}\left(g_{i}\right)-v_{i}\left(b_{i}\right)\right)$ for arbitrary fixed $g_{i}, b_{i}$. Here $w_{i}$ has no special meaning or interpretation. In particular, the normalized $w_{i} \mathrm{~s}$, 
while probabilities in a formal sense, are only mathematical devices and have no natural interpretation. The problem of nonidentifiability of probability under state-dependent expected utility has been discussed extensively; see Aumann (1971), Drèze (1987, Ch. 2), Kadane and Winkler (1988), Karni (1996), Karni and Schmeidler (1993), Karni and Mongin (2000, p. 233), Nau (1995), and Schervish et al. (1990).

A method of using constant-utility outcomes to identify probability in state-dependent expected utility by fixing two outcomes with state-independent outcomes, similar to Example 4.4, was used by Drèze (1987, Ch. 2 through idempotent acts), Maher (1993, Ch. 8), Karni (1993), and, finally, Karni (1999), with $b$ the worst and $g$ the best outcome. Karni and Schmeidler (1993, the Proposition) used a similar idea, with $b=0$ and $g$ infinitesimally close to $b$ through the requirement of identical derivatives $\lim _{g \rightarrow 0}\left(v_{i}(g)-\right.$ $\left.v_{i}(0)\right) /(g-0)$ at $b=0$. It is interesting to note that a technique used in state-dependent expected utility to measure subjective probabilities is formally similar to a convention used in the health domain to measure quality of life.

\section{Overlapping Attribute Sets}

The previous sections did not assume relations between attribute levels for different attributes. In many contexts, however, such relations are present. In intertemporal preferences the same commodities can be consumed at different time points, and in decision under uncertainty an outcome can be received under different events; see Example 4.5. This section considers identical attribute sets for different attributes, and examines the implications for the measurement of attribute utilities. The following observation, still formulated for a general setup with different attribute sets, is preparatory. It readily follows from Theorem 3.3.

Observation 5.1. For a set $A_{i} \subset X_{i}$ of anchor levels, preferences between prospects over the set $\left\{a_{i} x: a_{i} \in\right.$ $\left.A_{i}\right\}$ are independent of $x$. In particular, the utility $u_{i}\left(a_{i}, x\right)$ in Equations (4.3)-(4.5) is independent of $x$ whenever, besides $b_{i} \in A_{i}$ and $g_{i} \in A_{i}$, also $a_{i} \in A_{i}$.

The condition in the observation is reminiscent of utility independence. The condition is too weak to characterize anchor levels because it does not exclude scale differences between $U\left(a_{i}, x\right)$ and $U\left(a_{i}, y\right)$ for $x \neq y$ (Example 10.1(i) in the appendix). The rest of this section assumes that $X_{1}=\cdots=X_{n}=\mathscr{b}$ for a set $\mathscr{b}$ called the outcome set. For outcomes $\alpha, \beta$, we write $\alpha \succcurlyeq \beta$ if $(\alpha, \ldots, \alpha) \succcurlyeq(\beta, \ldots, \beta)$. We use the notation $\alpha_{i} x$ in analogy to $y_{i} x$; that is, $\alpha_{i} x$ denotes $x$ with $x_{i}$ replaced by $\alpha$.

In the setup of this section, outcomes can serve as anchor levels for different attributes. It is plausible that their utilities are then invariant over the various attributes. A formalization is as follows, where attribute $i$ is null if $\alpha_{i} x \sim \beta_{i} x$ for all $\alpha, \beta$, and $x$ and nonnull otherwise. $A \subset \mathscr{C}$ is a set of attribute-independent anchor levels if: (a) $A$ is a set of anchor levels for each attribute; (b) a preference $\alpha_{i} x \succcurlyeq\left(p: \gamma_{i} x ; 1-p: \beta_{i} x\right)$ is not only independent of $x$ (Observation 5.1), but also independent of $i$ for all nonnull attributes $i$ and for all $\alpha, \beta, \gamma \in A$. Example 10.1 shows that conditions (a) and (b) are logically independent.

For attribute-independent anchor levels $\beta, \gamma$, a strict preference $\gamma_{i} x \succ \beta_{i} x$ for some $i$ implies that such a strict preference holds for all nonnull $i$, and implies $\gamma \succ \beta$ as soon as there is at least one nonnull attribute. In that case, $\gamma_{i} x \succ \beta_{i} x$ for some nonnull $i$ if and only if the same holds for all nonnull $i$, which holds if and only if $\gamma>\beta$.

Observation 5.2. Assume that $\mathscr{b}=X_{1}=\cdots=X_{n}$ and that $A \subset \mathscr{C}$ is a set of attribute-independent anchor levels. Then:

(i) The preferences between prospects over the set $\left\{\alpha_{i} x: \alpha \in A\right\}$ are independent of $x$ and $i$ for all nonnull $i$.

(ii) On $A$, the functions $v_{i}, i=1, \ldots, n$ of Corollary 3.4 are the same up to unit and location.

(iii) For each $\alpha \in A$, the utility $u_{i}\left(\alpha_{i}, x\right)$ in Equations (4.3)-(4.5) is independent of $x$ and $i$ for all nonnull $i$ if, for attribute-independent anchor levels $\gamma \succ \beta$, $b_{i}=\beta$ and $g_{i}=\gamma$ for all such $i$.

If the set $A$ contains only two outcomes (or only two equivalence classes of outcomes), then attribute independence only requires that the two outcomes are ranked the same for each attribute. This requirement is not very restrictive or informative. For three or more equivalence classes, the condition is more restrictive and informative. The following theorem shows how overall utilities of alternatives can be related to utilities of attribute-independent anchor levels. 
Theorem 5.3. Assume that $\alpha, \beta$, and $\gamma$ are attributeindependent anchor levels, and that $i$ is nonnull. Then

(i) $\alpha_{i} x \sim\left(p: \gamma_{i} x ; 1-p: \beta_{i} x\right)$ if and only if $(\alpha, \ldots, \alpha) \sim$ $(p:(\gamma, \ldots, \gamma) ; 1-p:(\beta, \ldots, \beta))$.

(ii) If $\beta \prec \gamma$, and we scale $U(\beta, \ldots, \beta)=0$, $U(\gamma, \ldots, \gamma)=1, u_{i}(\beta, x)=0$, and $u_{i}(\gamma, x)=1$, then $U(\alpha, \ldots, \alpha)=u_{i}(\alpha, x)$.

Jansen et al. $(1998,2000)$ informally used a result as in (ii) above to relate the quality of life of chronic health states to the quality of life of temporary health states; see \$7. The following example illustrates the implications of attribute independence for anchor levels.

ExAMPle 5.4 (BehaVIoral State-INDEPENDENT Expected Utility). This example is like Example 4.5, with state-dependent expected utility and all attribute levels anchor levels. In addition, we assume that state-independent expected utility holds. That is, there exist probabilities $q_{i}$ and a function $u$ such that $U\left(x_{1}, \ldots, x_{n}\right)=\sum_{i=1}^{n} q_{i} u\left(x_{i}\right)$ is a subjective expected utility functional on $\mathscr{C}^{n}$. As will be demonstrated in Corollary 5.5(ii), such a representation exists if and only if all outcomes are attribute-independent anchor levels.

The probabilities $q_{i}$ are uniquely determined as soon as there are two or more nonindifferent attribute levels. In this case, fix arbitrary outcomes $g, b$ with $g \succ b$, to be used as attribute-independent anchor levels. Then $w_{i}=q_{i}(u(g)-u(b))$ for each $i$, and the normalized $w_{i} /\left(\sum_{j=1}^{n} w_{j}\right)$ are the subjective probabilities $q_{i}$. The probability $q_{i}$ can easily be inferred from preference through matching events with known probabilities, as follows: Receiving $g$ under state $i$ and $b$ otherwise is equivalent to receiving $g$ with objective probability $q_{i}$ and $b$ otherwise.

By Equations (4.3)-(4.5), we elicit $u_{i}(\alpha, x)=(u(\alpha)-$ $u(b)) /(u(g)-u(b))$, independently of $i$ and the $x_{j}$ s. The factor $u(g)-u(b)$ is a scaling factor that can be chosen arbitrarily; for instance, $u$ can be normalized so that this factor is one. Equations (4.3)-(4.5) elicit the traditional utility function $u$. In decision under uncertainty, we are often interested in the utilities of attribute levels, ${ }^{2}$ and acts are used only as a tool to measure those utilities.

\footnotetext{
${ }^{2}$ Remember that attributes refer to states of nature, and an attribute level designates an outcome in the traditional terminology, such as money or commodity bundle or health state.
}

\section{Corollary 5.5.}

(i) If $A \subset \mathscr{b}$ is a set of attribute-independent anchor levels, then there exist nonnegative $q_{1}, \ldots, q_{n}$ summing to one, and a function $u: A \rightarrow \mathbb{R}$, such that $U\left(x_{1}, \ldots, x_{n}\right)=$ $\sum_{j=1}^{n} q_{j} u\left(x_{j}\right)$ on $A^{n}$.

(ii) All outcomes (i.e., the whole set $\mathscr{C}$ ) are attributeindependent anchor levels if and only if subjective expected utility holds; i.e., the representation of (i) holds for all $\left(x_{1}, \ldots, x_{n}\right) \in \mathscr{C}^{n}$.

The result in (ii) is a generalization of the subjective expected-utility characterization of Anscombe and Aumann (1963). They used condition (i) of Observation 5.2 ("monotonicity"), whereas attribute independence is a weaker requirement that restricts monotonicity to particular one- and two-outcome prospects that are nondegenerate on all but one attribute (= "state").

The examples of decision under uncertainty that we have considered so far assumed no interactions, with all attribute levels (outcomes) anchor levels, sometimes attribute independent. The following example illustrates how our parameters can capture psychological interactions that violate separability.

Example 5.6 (Nonexpected Utility with DisapPOINTMENT). This example is like state-independent expected utility in Example 5.4, with $n=2, A=\mathscr{C}=$ $\{a, b, g, h\}, q_{1}=2 / 3, q_{2}=1 / 3$, and $u(a)=-1, u(b)=0$, $u(g)=1, u(h)=2$. There is, however, one modification of the function $U$. For act $(h, a)$, the agent will feel disappointment if the worst outcome $a$ obtains, given that the best outcome $h$ was more likely. Then the utility of $a$ is -4 instead of -1 , resulting in $U(h, a)$ $(=(2 / 3) 2+1 / 3(-4))=0$ instead of $U(h, a)=1$. For all other acts, $U$ is like the state-independent expected utility in Example 5.4. It is easy to verify that $\{b, g\}$ are attribute-independent anchor levels.

For eliciting utility from preference, we scale $u(b)=0$ and $u(g)=1$. Then,

$$
\begin{aligned}
(1:(b, b)) & \sim(1 / 2:(g, g) ; 1 / 2:(a, a)) \text { suggests that } \\
u(a) & =-1 .
\end{aligned}
$$

The observation $(1:(h, a)) \sim(1:(b, b))$, however, suggests factors beyond expected utility. Eliciting the factors of Equation (4.2) for $x=(h, a)$ and $i=2$, we obtain

- $w_{2}=U(h, g)-U(h, b)=(1 / 3)(u(g)-u(b))=$ $1 / 3=q_{2}$ 
- $V\left(x_{1}\right)=U(h, b)=2 u(h) / 3+u(b) / 3=4 / 3$;

- $u_{2}(a,(h, a))=(U(h, a)-U(h, b)) /(U(h, g)-$ $U(h, b))=(-4 / 3) /(1 / 3)=-4$.

We see that $u_{2}(a,(h, a))=-4$ deviates from $u(a)=-1$, which indeed reveals a violation of expected utility. In this manner, we can define and measure the interactions given a fixed outcome $h$ for the second state of nature.

As is common in decision theory, our formulas, based solely on observed choice, do not determine the psychological interpretations and background of the interactions. Our model captures all interactions through the utility $u_{2}(a,(h, a))$. Whether the interactions are due to disappointment while consuming $a$, and, even more basically, to what extent these interactions are at all realized during the consumption of $a$ in state 2 and not of $h$ in state 1 , should be based on extraneous information and interpretations from our part, which are to be determined by the context of application. In the application in \$7, for instance, we assume that an interaction takes place in the first attribute (the "period" of radiotherapy treatment), not on the basis of the mathematical preference model, but on the basis of medical and psychological arguments.

The representation of the above examples can be restated for other contexts, such as welfare evaluations, or intertemporal choice with health states specified for $n$ periods. Example 5.6 could concern the latter context, where it would be hard to adapt to health state $a$ if immediately following $h$, so that $(h, a)$ is evaluated especially negatively.

\section{History of Decision Analysis and Measurement Problems in the Health Domain}

The following two sections elaborate on Example 4.4. This section describes the history of decision analysis for QALY measurement, and some problems that arose there.

Until the 1970s, the most common measure for evaluating (heavy) medical treatments was the five-year survival rate, i.e., the proportion of patients still alive five years after a treatment. In the beginning of the 1980s, the usefulness of the more flexible expected utility criterion became understood in the medical domain (Weinstein and Stason 1977, McNeil et al. 1978). Keeney and Raiffa's (1976) techniques were subsequently used to justify specific evaluations of chronic health states. For example, $t$ years in health state $x$, followed by death, is evaluated by $w \times u(x)$, where $u$ measures quality of life and $w$ measures discounting (McNeil et al. 1981). Utility independence and other axioms justify this multiplicative evaluation (Pliskin et al. 1980).

The most popular way of evaluating the utility of nonchronic health outcomes is through QALYs (quality-adjusted life years); for surveys, see Fryback (1999) and Gold et al. (1996). Consider a general, nonchronic health profile of spending $t_{1}$ years in health state $x_{1}, t_{2}$ years in health state $x_{2}, \ldots$, and, finally, $t_{n}$ years in health state $x_{n}$, followed by death. The QALY utility of the health profile is $\sum_{j=1}^{n} w_{j} u\left(x_{j}\right)$, where $u\left(x_{j}\right)$ is the quality of life in health state $x_{j}$ and $w_{j}$ reflects the duration $\left(w_{j}=t_{j}\right.$ if zero discounting). For risky decisions, the expectation of this QALY utility is to be maximized.

With $G$ denoting good health and $D$ denoting death, the common scaling convention is $u(G)=1$ and $u(D)=0$. The quality of life $u\left(x_{j}\right)$ of a health state $x_{j}$ can be measured through the standard gamble method. For some fixed duration $t$, the probability $p$ is determined such that $(p,(t$ years $G), 1-p, D) \sim$ $\left(t\right.$ years $\left.x_{j}\right)$. Then $u\left(x_{j}\right)=p$ is set.

The QALY model is tractable, but requires many restrictive conditions. One of these conditions concerns the standard gamble method, which, like the other traditional methods, measures the quality of life of health states only when they are chronic. It is then assumed that the obtained quality-of-life measurements also apply to temporary health states. This assumption is especially problematic for health states that cannot even be conceived as chronic. For instance, undergoing a radiotherapy treatment (denoted $R$ ) for the rest of one's lifetime is not realistic.

To measure the quality of life of a temporary health state (say $R$, during $t_{1}$ years for $t_{1}=1 / 2$ ), Torrance (1986) proposed a chained method. In a first stage, for some properly chosen health states $A, B$, an equivalence is elicited such as $\left(t_{1}\right.$ years $R, t_{2}$ years $\left.G\right) \sim$ $\left(p,\left(t_{1}\right.\right.$ years $A, t_{2}$ years $\left.G\right) ; 1-p,\left(t_{1}\right.$ years $B, t_{2}$ years $\left.\left.G\right)\right)$. In the second stage, $u(A)$ and $u(B)$ are measured 
through traditional standard gamble questions, where $A$ and $B$ are taken as chronic. Finally, $u(R)=p u(A)+$ $(1-p) u(B)$ is set. $A$ and $B$ must be conceivable both as chronic and as temporary health states. Good health $(G)$ could be substituted for $A$, but death $(D)$ cannot realistically be substituted for $B$ if followed by $G$ at $t_{2}$. Torrance proposed using the worst temporary health state, other than death, for $B$.

Torrance (1986) emphasized that $A$ and $B$ should be conceivable, but did not specify preference conditions that these health states should satisfy. Jansen et al. $(1998,2000)$ and Johnston et al. (1998) informally stated some requirements. We first describe the domain of the studies by Jansen et al. These authors studied postoperative radiotherapy treatment for early-stage breast cancer patients. Radiotherapy reduces recurrences of breast cancer, but may induce undesirable side effects. To determine an optimal decision, the impact of side effects on the well-being of the patients has to be measured. Jansen et al. were particularly interested in the effect of experience with the treatment on the evaluation thereof. For the quality of life during a radiotherapy treatment, the psychological impact of the future prospects is all-decisive, and a measurement ignoring this impact is of no interest. The authors, therefore, decided to study radiotherapy only for a fixed period, followed by return to good health.

Jansen et al. $(1998,2000)$ emphasized that Torrance's temporary health states $A, B$ should be chosen with care, and used the term anchor health states for such proper choices. The anchor health states should be "broadly applicable and comparable across different contexts" (Jansen et al. 1998, p. 398), and "the utility of the anchor health state should not be systematically affected by its duration (utility independence) or by the health state following after (separability of preference)" (Jansen et al. 2000, p. 69). Johnston et al. (1998, p. 215) also suggested that there should be a "validity of the link [anchor] state." Like Torrance (1986), the latter authors used the worst temporary health state as one anchor state, and emphasized that the description of the good health state, which they used as another anchor state, should be unambiguous (p. 215). Borcherding et al. (1995, p. 24) suggested using intuitively meaningful anchor levels rather than maximal and minimal outcomes: "It might be more desirable to elicit meaningful anchors from the decision maker... and then to elicit weights for these ranges." The next section formalizes the conditions required for the anchor health states that were alluded to in the literature just cited.

\section{Anchor Health States for QALY Measurement with Intertemporal Dependencies}

This section describes the application of our technique to the problem studied by Jansen et al. $(1998,2000)$. Consider two attributes $(n=2)$. The first refers to an initial period of six months, the second to the rest of life. There are four health states:

\begin{tabular}{ccccc}
\hline Health state & Death & $\begin{array}{c}\text { Being in } \\
\text { hospital }\end{array}$ & Radiotherapy & Good health \\
\hline Abbreviation & $D$ & $B$ & $R$ & $G$ \\
\hline
\end{tabular}

For example, $(R, G)$ means radiotherapy in period 1 and good health in period 2. ${ }^{3}$ Figure 1 illustrates the domain of our study. We want to measure $u_{1}(R,(R, G))$, the well-being during radiotherapy if followed by good health.

We use the common scaling $U(G, G)=1$ and $U(D, D)=0$. The classical QALY model uses the points indicated by - in Figure 1 . It elicits the probability $p$ such that $(p,(G, G) ; 1-p,(D, D)) \sim(R, R)$ and then equates the chronic utility $U(R, R)=p$ with the temporary utility $u_{1}(R,(R, G))$. This approach is not satisfactory in this application because, first, the option $(R, R)$ is too unrealistic to be used in experiments, and second, if it could have been used, its utility $U(R, R)$ could not have been equated with $u_{1}(R,(R, G))$. An alternative would be to use the points indicated by $\downarrow$ in the figure. That is, $D$ and $G$ are anchor states for period 1 , we set ${ }^{4}$ $u_{1}(D,(R, G))=0$ and $u_{1}(G,(R, G))=1$, elicit $p$ such that $(p,(G, G) ; 1-p,(D, G)) \sim(R, G)$, and then equate $p$ with $u_{1}(R,(R, G))$. Unfortunately, this alternative approach fails because $(D, G)$ is an unrealistic option that cannot be used in experiments.

\footnotetext{
${ }^{3}$ More precisely, $R$ in period 1 designates a six-week radiotherapy treatment followed by four and a half months of possible side effects.

${ }^{4}$ Remember that $u_{1}(G,(R, G))$ denotes the utility of $G$ in period 1 in the context $(R, G)$, which means that $R$ is replaced there by $G$, so that $(G, G)$ results. The notation $u_{1}(D,(R, G))$ is similar.
} 
Figure 1 The Domain Study of Jansen et al. $(1998,2000)$

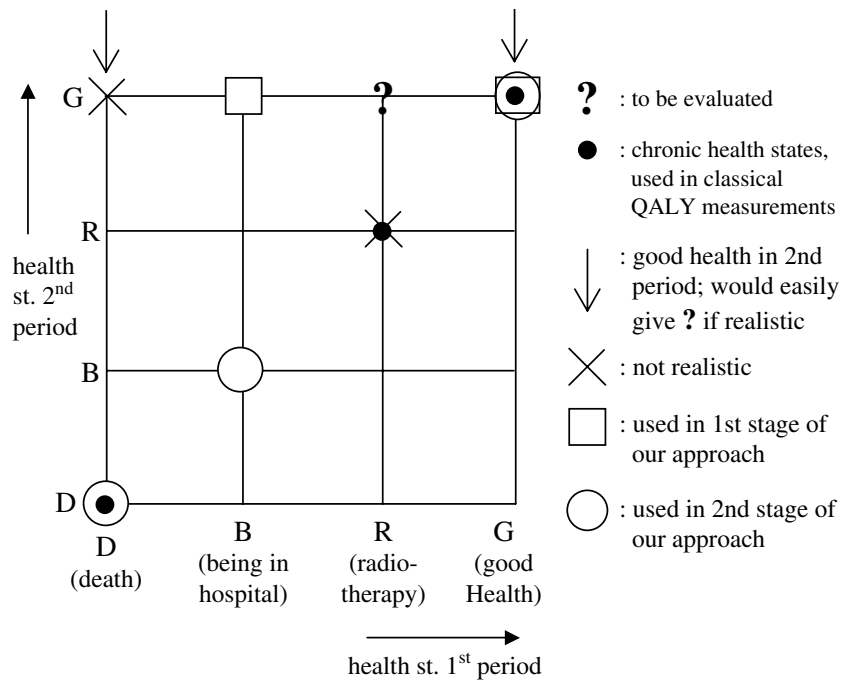

Jansen et al. $(1998,2000)$ used another anchor health state than $D$ for period 1, i.e., hospitalization caused by a serious accident $(B)$. Most people will be able to relate to this hypothetical health state. It is very distinct from the other health states considered in this experiment so as to minimize systematic interactions with those. In a first stage, the points indicated by $\square$ in Figure 1 were used. The preferences $(B, G) \preccurlyeq$ $(R, G) \preccurlyeq(G, G)$ held for virtually all patients, so that Equation (4.2) could be used, and the probability $q$ was measured such that

$$
(R, G) \sim(q,(G, G) ; 1-q,(B, G)) .
$$

In the notation of $\S 4$, which would entail a scaling $u_{1}(G,(R, G))=1$ and $u_{1}(B,(R, G))=0$, the result would be $u_{1}(R,(R, G))=q$.

The authors, however, wanted to follow the common scaling convention in the health domain that assigns quality of life zero to death and quality of life one to good health; i.e., $u_{1}(G,(R, G))=1$ and $u_{1}(D,(R, G))=0$. This scaling convention is important because it allows for comparisons of effects across different studies with different people and treatments. In the terminology of this paper, the common scaling convention assumes that $D$ and $G$ are attribute-independent anchor levels. The rescaling requires that $u_{1}(B,(R, G))$ be related to $u_{1}(G,(R, G))$ and $u_{1}(D,(R, G))$. To this effect, Jansen et al. used the points indicated by $\bigcirc$ in the figure in a second stage.
They elicited the utility of $B$ through the indifference

$$
(B, B) \sim(r,(G, G) ; 1-r,(D, D)) .
$$

Such an indifference implies $U(B, B)=r$. Given $U(D, D)=0=u_{1}(D,(R, G)), U(G, G)=1=u_{1}(G$, $(R, G))$, the authors assumed

$$
u_{1}(B,(R, G))=U(B, B),
$$

implying that $u_{1}(B,(R, G))=r$. The crucial step in this reasoning, Equation (7.3), results from Theorem 5.3(ii) (for $i=1)$. Substituting $u_{1}(B,(R, G))=r$ in Equation (7.1) finally yields

$$
u_{1}(R,(R, G))=q+(1-q) r .
$$

This equation gives the utility of radiotherapy during six months, incorporating the dependency on the good health following it. It can be justified by our theory under the assumption that $D, G$, and $B$ are attribute-independent anchor levels. The underlying preference conditions have been described in the preceding sections.

It is well understood that the underlying preference conditions will not be satisfied to a perfect degree. There will be individual variations in the degree of approximation. The health state $B$ was developed to avoid systematic biases as much as possible. Our claim is not that state $B$ is a perfect anchor state and that all biases have been completely eliminated. We only claim that $B$ is a better approximation than health states that have been used traditionally. Our measurement procedure did not completely avoid all biases, but it avoided biases better than the classical procedures that have been used so far.

We finally draw some conclusions from the medical application described in this section. The complex and general formulas of $\S \S 4$ and 5 have allowed for an experimentally simple measurement of quality of life. All interactions between separate time periods have been respected, and only hypothetical scenarios that are easy to imagine for the patients were used. The implementability of the method was found to be satisfactory. Several biases that have been known to occur in other measurements, such as loss aversion, could be avoided (Jansen et al. 1998, p. 397). The measurement, therefore, agreed better with other 
measurements such as time tradeoffs (for a definition, see Gold et al. 1996). For further discussion of empirical and psychological aspects, and conclusions for the effects of experience with radiotherapy on the wellbeing during the treatment, see Jansen et al. (1998, 2000).

\section{Discussion}

The contributions of Theorems 4.2 and 4.3 do not lie in the representations

$$
\begin{gathered}
U(x)=w_{i} u_{i}\left(x_{i}, x\right)+V\left(x_{1}, \ldots, x_{i-1}, x_{i+1}, \ldots, x_{n}\right) \text { or } \\
\sum_{j=1}^{n} w_{j} u_{j}\left(x_{j}, x\right)+W(x)
\end{gathered}
$$

per se. These representations are completely general, and, therefore, without predictive power. The representations become meaningful only in combination with the second parts of the theorems, showing the empirical meaning of the parameters.

A utility difference $U\left(x_{j} z\right)-U\left(y_{j} z\right)$ can be measured by classical methods without resorting to anchor levels. For instance, if $U(h)=1, U(\ell)=0$, and $x_{j} z$ and $y_{j} z$ are between $h$ and $\ell$ in preference, then we can find $p$ and $q$ such that $x_{j} z \sim p h+(1-p) \ell$ and $y_{j} z \sim$ $q h+(1-q) \ell$. We then get

$$
U\left(x_{j} z\right)-U\left(y_{j} z\right)=p-q .
$$

Without anchor levels available, however, it is not easy to interpret such differences. They cannot be related to a representation with identifiable parameters, such as in Theorems 4.2 or 4.3. In addition, general measurements as in Equation (8.1) are not experimentally tractable if $h$ and $\ell$ are not related to the stimuli of the study (compare Jansen et al. 1998, 2000). Because of the absence of interpretations for Equation (8.1), such general equations are not useful in applications (Borcherding et al. 1995, pp. 9-10).

The elicitation of our model remains complex. Without further restrictions on the interactions, the measurement of $u_{j}\left(y_{j}, x\right)$ has to be redone for every separate $x$. Our result cannot simplify the preference system beyond its intrinsic complexity. The parameters, while complex, are, at least, well defined and identifiable.
Our technique of assuming some levels with independent utility and using them to measure utilities of other levels is reminiscent of a technique for measuring utility in Skiadas (1997). He considered decision under uncertainty with all kinds of interactions and violations of separability permitted. He assumed that there is a sufficiently rich set of canonical consequences such that acts taking only those consequences do satisfy separability and all expected utility axioms. Then a general act can be matched with a canonical act that is indifferent, conditional upon each state, and the expected utility of the general act can, thus, be determined.

\section{Conclusion}

This paper has proposed a new theoretical generalization of attribute independence, based on anchor levels. These are relatively stable levels of outcomes, and their values are unaffected by context and interactions. Anchor levels give alternative, more general, characterizations of classical representations such as additively decomposable multiattribute utility (Fishburn 1965) and subjective expected utility (Anscombe and Aumann 1963). They can characterize models that allow for general interactions between attributes, and they can make attribute utilities identifiable in such general models. Examples illustrated the meaning of our concepts and implications for the measurement of disappointment, of subjective probabilities if utility is state dependent, and of QALYs in the absence of temporal separability. The method was applied in a study of the well-being of patients during radiotherapy treatment.

A simple practical recommendation for the measurement of utility is as follows. No matter how complex the interaction between attributes is, attribute utilities can be defined and measured if anchor levels can be constructed for these attributes. We hope that this paper, advancing a very general mathematical theory but originating from a simplification of empirical measurements, has demonstrated the usefulness of anchor levels for multiattribute utility theory.

\section{Appendix A. Proofs}

Proof of THeOREM 3.3. If $U$ is of the form in the theorem, then for all $g_{i}, b_{i} \in A_{i}, U\left(g_{i} y\right)-U\left(b_{i} y\right)=v_{i}\left(g_{i}\right)-$ $v_{i}\left(b_{i}\right)$ is indeed independent of $y$. Hence, $A_{i}$ is a set of anchor levels. Conversely, assume that $U\left(g_{i} x\right)-U\left(b_{i} x\right)$ is 
independent of $x$ for all $g_{i}, b_{i} \in A_{i}$. Fix any $r=\left(r_{1}, \ldots, r_{n}\right)$ with $r_{i} \in A_{i}$, and write, for any $x$ with $x_{i} \in A_{i}, U(x)=U(x)-$ $U\left(r_{i} x\right)+U\left(r_{i} x\right)=U\left(x_{i} r\right)-U(r)+U\left(r_{i} x\right)$. Define $v_{i}\left(x_{i}\right)=$ $U\left(x_{i} r\right)-U(r)$ and $V\left(x_{1}, \ldots, x_{i-1}, x_{i+1}, \ldots, x_{n}\right)=U\left(r_{i} x\right)$.

Proof of Corollary 3.4. Statement (i) follows immediately from Theorem 3.3. For statement (ii), the proof of Theorem 3.3 is first applied to $i=1$, and then proceeds inductively. Fix $x_{1}$ at any level, say $r_{1}$, then decompose $V\left(x_{2}, \ldots, x_{n}\right)$ as $v_{2}\left(x_{2}\right)+V^{*}\left(x_{3}, \ldots, x_{n}\right)$, etc.

Proof of Theorem 4.2. First assume that a decomposition of $U$ as described exists. Then, using independence of $V$ from the $i$ th attribute, $U\left(g_{i} x\right)-U\left(b_{i} x\right)=w_{i}\left(u_{i}\left(g_{i}\right)-\right.$ $\left.u_{i}\left(b_{i}\right)\right)=w_{i}$ (the latter by Equations (4.3)-(4.5)), which is indeed independent of $x$. By Equation (3.3), $\left\{g_{i}, b_{i}\right\}$ are anchor levels.

For the reversed implication, assume that $\left\{g_{i}, b_{i}\right\}$ are anchor levels. $w_{i}, u_{i}$, and $V$ are defined as in the beginning of $\S 4$; (i) and (ii) are satisfied. Because $w_{i}$ has to be $U\left(g_{i} x\right)-U\left(b_{i} x\right)$, it is unique up to the same scale factor as $U$. Similarly, $V$ is unique up to the same unit and location as $U$. Substitution of expected utility shows that $U(x)-$ $U\left(b_{i} x\right)$ is $w_{i} u_{i}\left(x_{i}, x\right)$ for each of the three cases described in Equations (4.3)-(4.5), which implies (iii). Uniqueness of $u_{i}$ follows immediately from Equations (4.3)-(4.5) and, thus, all uniqueness results have been established.

Proof of Theorem 4.3. Note that the strict preferences assumed in the beginning of the theorem imply that all attributes affect preferences. First assume the decomposition described in the theorem. Then, by (ii),

$$
U\left(g_{i} x\right)-U\left(b_{i} x\right)=w_{i}\left(u_{i}\left(g_{i}, x\right)-u_{i}\left(b_{i}, x\right)\right)=w_{i}
$$

(the latter by (ii)), which is independent of $x$. By Equation (3.3), $\left\{g_{i}, b_{i}\right\}$ are anchor levels.

Next, assume that all $\left\{g_{i}, b_{i}\right\}$ are anchor levels. Define $w_{i}=U\left(g_{i} x\right)-U\left(b_{i} x\right)$, which, by Equation (3.3), is independent of $x$ and positive because $U\left(g_{i} b\right)-U(b)$ is positive. Hence, (i) holds. Define $u_{i}\left(x_{i}, x\right)$ as in Equations (4.3)-(4.5) so that (ii) is satisfied, and

$$
U(x)-U\left(b_{i} x\right)=u_{i}\left(x_{i}, x\right)\left(U\left(g_{i} x\right)-U\left(b_{i} x\right)\right) .
$$

We define

$$
W(x)=\sum_{j=1}^{n} U\left(b_{j} x\right)-(n-1) U(x) .
$$

To establish the form of $U$, we have

$$
\begin{aligned}
U(x) & =\sum_{j=1}^{n}\left(U(x)-U\left(b_{j} x\right)\right)-(n-1) U(x)+\sum_{j=1}^{n} U\left(b_{j} x\right) \\
& =\sum_{j=1}^{n}\left(U(x)-U\left(b_{j} x\right)\right)+W(x) \\
& =\sum_{j=1}^{n} u_{j}\left(x_{j}, x\right)\left(U\left(g_{j} x\right)-U\left(b_{j} x\right)\right)+W(x) \\
& =\sum_{j=1}^{n} u_{j}\left(x_{j}, x\right) w_{j}+W(x) .
\end{aligned}
$$

This establishes the form of $U$. For (iii), we have

$$
\begin{aligned}
\sum_{j \neq i} w_{j} u_{j}\left(x_{j}, x\right)+W(x) & \\
= & \sum_{j \neq i} u_{j}\left(x_{j}, x\right)\left(U\left(g_{j} x\right)-U\left(b_{j} x\right)\right)+W(x) \\
= & \sum_{j \neq i}\left(U(x)-U\left(b_{j} x\right)\right)+W(x) \\
= & \sum_{j \neq i}\left(U(x)-U\left(b_{j} x\right)\right)+\sum_{j=1}^{n} U\left(b_{j} x\right)-(n-1) U(x) \\
= & U\left(b_{i} x\right),
\end{aligned}
$$

which is independent of the $i$ th attribute level $x_{i}$, establishing the second part of (iii). The equivalence of the first and second part of (iii) follows from substitution of the formula for $U$, both parts being equivalent to

$$
\sum_{j \neq i} w_{j} u_{j}\left(x_{j}, y_{i} x\right)+W\left(y_{i} x\right)-\sum_{j \neq i} w_{j} u_{j}\left(x_{j}, z_{i} x\right)-W\left(z_{i} x\right)=0 .
$$

The uniqueness results follow from Theorem 4.2. Let us add that, by the definition of the $w_{j} s$,

$$
\sum_{j=1}^{n} w_{j}=U(g)-U(b) .
$$

Proof of Observation 5.2. If $A$ does not contain more than one $\succcurlyeq$ indifference class, then for all $x, i,\left\{\alpha_{i} x: \alpha \in A\right\}$ is contained in one indifference class. All preferences between prospects over the set are, therefore, indifferences and, thus, are obviously independent of $x$ and $i$. Then the first two claims of the observation hold. Outcomes $\beta, \gamma$ as assumed in the last claim of the observation then do not exist and, hence, this claim is vacuously satisfied.

We assume henceforth that $A$ contains two outcomes $\gamma \succ \beta$. A preference $\gamma_{i} x \succ \beta_{i} x$ is independent of $x$ and $i$, for all $\beta, \gamma \in A$ and nonnull $i$. This independence implies that the same $\beta=b_{i}$ and $\gamma=g_{i}$ can be used for all nonnull $i$ in Equations (4.3)-(4.5). By attribute independence, the utility function $u_{i}(\cdot, x)$ in Equations (4.3)-(4.5) is independent of $x$ and $i$ if $i$ is nonnull. This implies claim (iii) of the observation. For each $x$ and nonnull $i$, preferences over prospects over the set $\left\{\alpha_{i} x: \alpha \in A\right\}$ can be represented by expected utility with the utility function defined in Equations (4.3)-(4.5). This implies claim (i) of the observation. It also implies claim (ii) of the observation by the usual uniquess conditions of expected utility.

Proof of Theorem 5.3. We first derive (i). Nonnull attributes do not affect preferences and can be ignored. We suppress them in this proof, and assume that all attributes are nonnull. The constant alternative $(\alpha, \ldots \alpha)$ is denoted by $\bar{\alpha}$.

Assume $i=1$. Let $\alpha_{1} x \sim\left(p: \gamma_{1} x ; 1-p: \beta_{1} x\right)$ for attributeindependent anchor levels $\alpha, \beta, \gamma$, and some $x$. We first prove that $\bar{\alpha} \sim(p: \bar{\gamma} ; 1-p: \bar{\beta})$, and this will constitute the 
major part of the proof. Because $\{\alpha, \beta, \gamma\}$ are attributeindependent anchor levels,

$$
\alpha_{i} y \sim\left(p: \gamma_{i} y ; 1-p: \beta_{i} y\right) \text { for all } y, i
$$

We proceed by induction. Let $\bar{\gamma}^{i}$ denote the alternative $(\gamma, \ldots, \gamma, \alpha, \ldots, \alpha)$ with the first $i$ attributes equal to $\gamma$ and the remaining attributes equal to $\alpha$. Similarly, $\bar{\beta}^{i}$ denotes the alternative $(\beta, \ldots, \beta, \alpha, \ldots, \alpha)$ with the first $i$ attributes equal to $\beta$ and the remaining attributes equal to $\alpha$.

$$
i \text { th induction hypothesis: } \bar{\alpha} \sim\left(p: \bar{\gamma}^{i} ; 1-p: \bar{\beta}^{i}\right) \text {. }
$$

For $i=1$, the induction hypothesis follows immediately from Equation (10.2), with $i=1$ and $y=\bar{\alpha}$. Assume that the hypothesis has been proved for some $i$. We derive it for $i+1$, with the following numbered indifferences and equalities explained thereafter and $(i+1)$ th attributes denoted in bold printing.

$$
\begin{aligned}
\bar{\alpha} \stackrel{1}{\sim}\left(p: \bar{\gamma}^{i} ; 1-p: \bar{\beta}^{i}\right)=\left(p: \boldsymbol{\alpha}_{i+1} \bar{\gamma}^{i} ; 1-p: \boldsymbol{\alpha}_{i+1} \bar{\beta}^{i}\right) \\
\stackrel{2}{\sim}\left(p:\left(p: \boldsymbol{\gamma}_{i+1} \bar{\gamma}^{i} ;(1-p): \boldsymbol{\beta}_{i+1} \bar{\gamma}^{i}\right) ;\right. \\
\left.\quad 1-p:\left(p: \boldsymbol{\gamma}_{i+1} \overline{\boldsymbol{\beta}}^{i} ;(1-p): \boldsymbol{\beta}_{i+1} \overline{\boldsymbol{\beta}}^{i}\right)\right) \\
\stackrel{3}{=}\left(p^{2}: \boldsymbol{\gamma}_{i+1} \bar{\gamma}^{i} ; p(1-p): \boldsymbol{\beta}_{i+1} \bar{\gamma}^{i} ;\right. \\
\left.\quad \quad(1-p): \boldsymbol{\gamma}_{i+1} \bar{\beta}^{i} ;(1-p)^{2}: \boldsymbol{\beta}_{i+1} \bar{\beta}^{i}\right) \\
\stackrel{4}{\sim}\left(p^{2}: \boldsymbol{\gamma}_{i+1} \bar{\gamma}^{i} ; p(1-p): \boldsymbol{\gamma}_{i+1} \bar{\gamma}^{i} ; p(1-p): \boldsymbol{\beta}_{i+1} \bar{\beta}^{i} ;(1-p)^{2}: \boldsymbol{\beta}_{i+1} \bar{\beta}^{i}\right) \\
=\left(p: \boldsymbol{\gamma}_{i+1} \bar{\gamma}^{i} ; 1-p: \boldsymbol{\beta}_{i+1} \bar{\beta}^{i}\right) \\
=\left(p: \bar{\gamma}^{i+1} ; 1-p: \bar{\beta}^{i+1}\right) . \\
\stackrel{1}{\sim}: \text { The induction hypothesis. } \\
\stackrel{2}{\sim} \text { and } \stackrel{3}{=}: \text { Substituting } \bar{\gamma}^{i} \text { and } \bar{\beta}^{i} \text { for } y \text { in Equation (10.2) }
\end{aligned}
$$
gives

$$
\begin{aligned}
& \bar{\gamma}^{i}=\boldsymbol{\alpha}_{i+1} \bar{\gamma}^{i} \sim\left(p: \boldsymbol{\gamma}_{i+1} \bar{\gamma}^{i} ;(1-p): \boldsymbol{\beta}_{i+1} \bar{\gamma}^{i}\right) \quad \text { and } \\
& \bar{\beta}^{i}=\boldsymbol{\alpha}_{i+1} \bar{\beta}^{i} \sim\left(p: \boldsymbol{\gamma}_{i+1} \bar{\beta}^{i} ;(1-p): \boldsymbol{\beta}_{i+1} \bar{\beta}^{i}\right) .
\end{aligned}
$$

In expected utility under risk, replacing an alternative $\left(\bar{\gamma}^{i}\right.$ or $\left.\bar{\beta}^{i}\right)$ in a prospect by an indifferent prospect

$$
\left(\left(p: \boldsymbol{\gamma}_{i+1} \bar{\gamma}^{i} ;(1-p): \boldsymbol{\beta}_{i+1} \bar{\gamma}^{i}\right) \text { or }\left(p: \boldsymbol{\gamma}_{i+1} \bar{\beta}^{i} ;(1-p): \boldsymbol{\beta}_{i+1} \bar{\beta}^{i}\right)\right)
$$

and multiplying out the probabilities leads to an indifferent new prospect. In this manner, we substitute the two preceding indifferences, with the two-stage-prospect notation following $\stackrel{2}{\sim}$ only a shorthand notation for the corresponding one-stage prospect (two-stage prospects are not considered in our formal model).

$\stackrel{4}{\sim}$ : Because $\beta$ and $\gamma$ are anchor levels for attribute $i+1$, we may, by Equation (3.3) and expected utility, exchange these attributes in the (equally likely!) second and third alternatives in the prospect to obtain the indifferent prospect following $\stackrel{4}{\sim}$. The induction hypothesis has been established for $i+1$. Finally, it follows for $i=n$; i.e.,

$$
\bar{\alpha} \sim\left(p: \bar{\gamma}^{n} ; 1-p: \bar{\beta}^{n}\right)=(p: \bar{\gamma} ; 1-p: \bar{\beta})
$$

The implication

$$
\alpha_{1} x \sim\left(p: \gamma_{1} x ; 1-p: \beta_{1} x\right) \quad \Rightarrow \quad \bar{\alpha} \sim(p: \bar{\gamma} ; 1-p: \bar{\beta})
$$

has been established.

To complete the proof of the logical equivalence claimed in statement (i) of the theorem, a number of cases are distinguished. First, assume that $\gamma_{1} x \succ \beta_{1} x$. Then,

$$
\begin{aligned}
(\gamma, \gamma, \ldots, \gamma) & \succ(\beta, \gamma, \ldots, \gamma) \succ(\beta, \beta, \gamma, \ldots, \gamma) \\
& \succ \cdots \succ(\beta, \ldots, \beta, \gamma) \succ(\beta, \ldots, \beta, \beta)
\end{aligned}
$$

i.e., $\bar{\gamma} \succ \bar{\beta}$. The probabilities $p$ and $p^{\prime}$ in both

$$
\alpha_{i} x \sim\left(p: \gamma_{i} x ; 1-p: \beta_{i} x\right) \text { and } \bar{\alpha} \sim\left(p^{\prime}: \bar{\gamma} ; 1-p^{\prime}: \bar{\beta}\right)
$$

are uniquely determined, and the first part of the proof implies that $p=p^{\prime}$. In this case, the logical equivalence in the theorem holds. It similarly does if $\gamma_{1} x \prec \beta_{1} x$. Finally, we consider the case $\gamma_{1} x \sim \beta_{1} x$. Then

$$
\begin{aligned}
(\gamma, \gamma, \ldots, \gamma) & \sim(\beta, \gamma, \ldots, \gamma) \sim(\beta, \beta, \gamma, \ldots, \gamma) \\
& \sim \ldots \sim(\beta, \ldots, \beta, \gamma) \sim(\beta, \ldots, \beta, \beta),
\end{aligned}
$$

i.e., $\bar{\gamma} \sim \bar{\beta}$. Hence,

$$
\alpha_{i} x \sim\left(p: \gamma_{i} x ; 1-p: \beta_{i} x\right) \text { and } \bar{\alpha} \sim\left(p^{\prime}: \bar{\gamma} ; 1-p^{\prime}: \bar{\beta}\right)
$$

hold for all probabilities $p, p^{\prime}$. Again, the logical equivalence in statement $(i)$ in the theorem holds.

If $\beta \preccurlyeq \alpha \preccurlyeq \gamma$, then statement (ii) follows from statement (i) by substitution. Other cases follow by exchanging the variables $\alpha, \beta$, and $\gamma$ and renormalizing utility.

Proof of Corollary 5.5.

(i) The result is trivial if $c_{i} x \sim d_{i} x$ for all $i, x, c_{i}, d_{i} \in A$; then $U$ is constant on $A^{n}$ and $u$ is the same constant on $A$, and the $q_{i}$ s can be chosen arbitrarily. Suppose, therefore, that $g_{i} x \succ b_{i} x$ for some $i, x$, and $g, b$ in $A$. By attribute independence, $g_{i} x>b_{i} x$ for all $x$ and nonnull $i$, and $U(g, \ldots, g)>$ $U(b, \ldots, b)$ (the latter inequality follows because there is at least one nonnull $i)$. We may assume that $U(g, \ldots, g)=1$ and $U(b, \ldots, b)=0$ and use $\{g, b\}$ in Equations (4.3)-(4.5) for all $i$. For each $x$ and nonnull $i$, we can interpret $u_{i}\left(\alpha_{i}, x\right)$ as a von Neumann-Morgenstern utility function for prospects over the set $\left\{\alpha_{i} x: \alpha \in A\right\}$.

By Corollary 3.4(ii) applied to the restriction of $\succcurlyeq$ to the prospects over $A^{n}, U\left(a_{1}, \ldots, a_{n}\right)$ is additively decomposable as $\sum_{j=1}^{n} v_{j}\left(a_{j}\right)$ (where we write $v_{j}$ for the $w_{j} u_{j}$ of Equation (2.1)) on $A^{n}$. We may assume that $v_{j}(b)=0$ for all $j$. For all nonnull $i$,

$$
u_{i}\left(a_{i}, x\right)=\left(v_{i}\left(a_{i}\right)-v_{i}(b)\right) /\left(v_{i}(g)-v_{i}(b)\right)=v_{i}\left(a_{i}\right) / v_{i}(g)
$$

on $A$. By Observation 5.2(ii), the $u_{i}(\cdot, x) \mathrm{s}$ (for all $i$ and $x$ ) when restricted to $A$ are the same up to unit and location; hence, so are the $v_{j}$ s. Because the $u_{i}(\cdot, x)$ s are 0 at $b$ and 1 at $g$ for all nonnull $i$, they must be identical on $A$. Define $u=u_{i}$ 
for any nonnull $i$, and $q_{i}=w_{i}=U\left(g_{i} x\right)-U\left(b_{i} x\right)=v_{i}(g)$ for each $i$. Now $v_{i}=q_{i} u$, also for null $i$ (that have $q_{i}=0$ ), and the subjective expected utility representation follows.

(ii) The only-if part follows immediately from (i), and the if part follows from substitution.

EXAMPLE 10.1 (INDEPENDENCE OF CONDITIONS (A) AND (B) IN THE DEFINITION OF ATtRIBUTE-INDEPENDENT ANCHOR LEVELS).

(i) $U\left(x_{1}, x_{2}\right)=x_{1} x_{2}$ on $\mathbb{R}_{++}^{2}$ satisfies condition (b), with $\alpha_{i} x \succcurlyeq\left(p: \gamma_{i} x ; 1-p: \beta_{i} x\right)$ if and only if $\alpha \geq p \gamma+(1-p) \beta$ independently of $i$ (all nonnull) and $x$ indeed, but there are no nontrivial anchor levels.

(ii) For $U\left(x_{1}, x_{2}\right)=x_{1}+\exp \left(x_{2}\right)$ on $\mathbb{R}_{++}^{2}$, all attribute levels are anchor levels (condition (a)), but the preference between $\alpha_{i} x$ and $\left(p: \gamma_{i} x ; 1-p: \beta_{i} x\right)$ depends on $i$, with risk neutrality for $i=1$ and strict risk seeking for $i=2$, so that condition (b) is violated for all sets of three or more attribute levels.

\section{References}

Anscombe, F. J., R. J. Aumann. 1963. A definition of subjective probability. Ann. Math. Statist. 34 199-205.

Arrow, Kenneth J. 1951. Alternative approaches to the theory of choice in risk-taking situations. Econometrica 19 404-437.

Aumann, Robert J. 1971. Letter from Robert Aumann to Leonard Savage. Jacques H. Drèze, ed. Essays on Economic Decision under Uncertainty. Cambridge University Press, Cambridge, U.K.

Becker, Gary S. 1996. Accounting for Tastes. Harvard University Press, Cambridge, MA.

Bleichrodt, Han, John Miyamoto. 2003. A characterization of quality-adjusted life-years under cumulative prospect theory. Math. Oper. Res. 28 181-193.

Borcherding, Katrin, Stefanie Schmeer, Martin Weber. 1995. Biases in multiattribute weight elicitation. Jean-Paul Caverni, Maya Bar-Hillel, F. Hutton Barron, Helmut Jungermann, eds. Contributions to Decision Making-I, Elsevier, Amsterdam, The Netherlands, 3-28.

Broome, John R. 1993. Qalys. J. Public Econom. 50 149-167.

de Finetti, Bruno. 1932. Sulla Preferibilità. Giornale Econom. Ann. Econom. 11 685-709.

Dolan, Paul. 2000. The measurement of health-related quality of life for use in resource allocation decisions in health care. Antony J. Culyer, Joseph P. Newhouse, eds. Handbook of Health Economics. Elsevier, Amsterdam, The Netherlands, 1723-1760.

Dolan, Paul, Claire Gudex. 1995. Time preference, duration and health state valuations. Health Econom. 4 289-299.

Drèze, Jacques H. 1987. Essays on Economic Decision Under Uncertainty. Cambridge University Press, Cambridge, U.K.

Dyckerhoff, Rainer. 1994. Decomposition of multivariate utility functions in non-additive expected utility theory. J. MultiCriteria Decision Anal. 3 41-58.

Dyer, James S., Rakesh K. Sarin. 1982 Relative risk aversion. Management Sci. 28 875-886.

Ellsberg, Daniel. 1954. Classic and current notions of measurable utility. Econom. J. 62 528-556.

Farquhar, Peter H., Peter C. Fishburn. 1981. Equivalence and continuity in multivalent preference structures. Oper. Res. 29 282-293.
Fishburn, Peter C. 1965. Independence in utility theory with whole product sets. Oper. Res. 13 28-45.

Fishburn, Peter C., Irving H. LaValle. 1992. Multiattribute expected utility without the Archimedean axiom. J. Math. Psych. 36 573-591.

Fryback, Dennis G. 1999. The QALY model: Utilities for cost-utility analysis in health care. James C. Shanteau, Barbara A. Mellers, David A. Schum, eds. Decision Science and Technology: Reflections on the Contributions of Ward Edwards. Kluwer, Dordrecht, The Netherlands, 331-351.

Fryback, Dennis G., Ralph L. Keeney. 1983. Constructing a complex judgmental model: An index of trauma severity. Management Sci. 29 869-883.

Gold, Marthe R., Joanna E. Siegel, Louise B. Russell, Milton C. Weinstein. 1996. Cost-Effectiveness in Health and Medicine. Oxford University Press, New York.

Jansen, Sylvia J. T., Anne M. Stiggelbout, Peter P. Wakker, Marianne A. Nooij, Evert M. Noordijk, Job Kievit. 2000. Unstable preferences: A shift in valuation or an effect of the elicitation procedure. Medical Decision Making 20 62-71.

Jansen, Sylvia J. T., Anne M. Stiggelbout, Peter P. Wakker, Thea P. M. Vliet Vlieland, Jan-Willem H. Leer, Marianne A. Nooy, Job Kievit. 1998. Patient utilities for cancer treatments: A study on the feasibility of a chained procedure for the standard gamble and time tradeoff. Medical Decision Making 18 391-399.

Johannesson, Magnus, Bengt Jönsson, Goran Karlsson. 1996. Outcome measurement in economic evaluation. Health economics. Health Econom. 5 279-296.

Johnston, Katharine, Jackie Brown, Karen Gerard, Moira O'Hanlon, Alison Morton. 1998. Valuing temporary and chronic health states associated with breast screening. Soc. Sci. Medicine 47 213-222.

Kadane, Joseph B., Robert L. Winkler. 1988. Separating probability elicitation from utilities. J. Amer. Statist. Assoc. 83 357-363.

Kahneman, Daniel. 1994. New challenges to the rationality assumption. J. Institut. Theoret. Econom. 150 18-36.

Karni, Edi. 1993. Subjective expected utility with state-dependent preferences. J. Econom. Theory 60 428-438.

Karni, Edi. 1996. Probabilities and beliefs. J. Risk Uncertainty 13 249-262.

Karni, Edi. 1999. Elicitation of subjective probabilities when preferences are state-dependent. Internat. Econom. Rev. 40 479-486.

Karni, Edi, Philippe Mongin. 2000. On the determination of subjective probability by choices. Management Sci. 46 233-248.

Karni, Edi, David Schmeidler. 1993. On the uniqueness of subjective probabilities. Econom. Theory 3 267-277.

Keeney, Ralph L. 1992 Value-Focused Thinking. Harvard University Press, Cambridge, MA.

Keeney, Ralph L., Howard Raiffa. 1976. Decisions with Multiple Objectives. Wiley, New York (2nd ed. 1993, Cambridge University Press, Cambridge, U.K.).

Krabbe, Paul F. M., Gouke J. Bonsel. 1998. Sequence effects, health profiles, and the QALY model: In search of realistic modeling. Medical Decision Making 18 178-186.

Kupperman, M., Stephen C. Shiboski, David H. Feeny, M. E. P. Elkin, M. A. E. Washington. 1997. Can preference scores for discrete states be used to derive preference scores for an entire path of events? An application to prenatal diagnosis. Medical Decision Making 42 42-55. 
Leontief, Wassily W. 1947. A note on the interrelation of subsets of independent variables of a continuous function with continuous first derivatives. Bull. Amer. Math. Soc. 53 343-350.

Loewenstein, George F., John Elster. 1992. Choice over Time. Russell Sage Foundation, New York.

Loomes, Graham, Linda McKenzie. 1989. The use of QALYs in health care decision making. Soc. Sci. Medicine 28 299-308.

Maher, Patrick. 1993. Betting on Theories. Cambridge University Press, Cambridge, U.K.

McDaniels, Timothy L. 1995. Using judgment in resource management: A multiple objective analysis of a fisheries management decision. Oper. Res. 43 415-426.

McNeil, Barbara J., Ralph Weichselbaum, Stephen G. Pauker. 1978. Fallacy of the five-year survival in lung cancer. New England J. Medicine 299 1397-1401.

McNeil, Barbara J., Ralph Weichselbaum, Stephen G. Pauker. 1981. Speech and survival: Tradeoffs between quality and quantity of life in laryngeal cancer. New England J. Medicine 305 982-987.

Miyamoto, John M., Stephen A. Eraker. 1988. A multiplicative model of the utility of survival duration and health quality. J. Experiment. Psych. General 117 3-20.

Nau, Robert F. 1995. Coherent decision analysis with inseparable probabilities and utilities. J. Risk Uncertainty 10 71-91.

$\mathrm{Ng}$, Yew-Kwang. 1999. Utility, informed preference, or happiness: Following Harsanyi's argument to its logical conclusion. Soc. Choice Welfare 16 197-216.

Payne, John W., Dan J. Laughhunn, Roy L. Crum. 1984. An experimental study of multiattribute risky choice. Management Sci. $\mathbf{3 0}$ 1350-1361.

Pliskin, Joseph S., Donald S. Shepard, Milton C. Weinstein. 1980. Utility functions for life years and health status. Oper. Res. 28 206-224.

Prelec, Drazen, George F. Loewenstein. 1991. Decision making over time and under uncertainty: A common approach. Management Sci. 37 770-786.

Richard, S. F. 1975. Multivariate risk aversion, utility independence, and separable utility functions. Management Sci. 22 12-21.

Richardson, Jeff, Jane Hall, Glenn Salkfeld. 1996. The measurement of utility in multiphase health states. Internat. J. Tech. Assessment Health Care 13 35-48.
Rubin, Herman. 1987. A weak system of axioms for "rational" behavior and the nonseparability of utility from prior. Statist. Decision 5 47-58.

Sackett, David L., George W. Torrance. 1978. The utility of different health states as perceived by the general public. J. Chronic Disease 31 697-704.

Savage, Leonard J. 1954. The Foundations of Statistics. Wiley, New York (2nd ed. 1972, Dover, New York).

Schervish, Mark J., Teddy Seidenfeld, Joseph B. Kadane. 1990. Statedependent utilities. J. Amer. Statist. Association 85 840-847.

Skiadas, Costis. 1997. Conditioning and aggregation of preferences. Econometrica 65 347-367.

Stiggelbout, Anne M., Hanneke C. J. M. de Haes. 2001. Patient preference for cancer therapy: An overview of measurement approaches. J. Clinical Oncology 19 220-230.

Stigler, George J. 1950. The development of utility theory: I; II. J. Political Econom. 58 307-327, 373-396.

Strotz, Robert H. 1957. The empirical implications of a utility tree. Econometrica 25 269-280.

Sutherland, Heather J., Hillary A. Llewelynn-Thomas, Norman F. Boyd, James E. Till. 1982. Attitudes toward quality of life: The concept of "maximal endurable time." Medical Decision Making 2 299-309.

Torrance, George W. 1986. Measurement of health state utilities for economic appraisal: A review. J. Health Econom. 5 1-30.

Torrance, George W. 1987. Utility approach to measuring healthrelated quality of life. J. Chronic Diseases 40 593-600.

Torrance, George W., David H. Feeny, William J. Furlong, Ronald D. Barr, Yuemin Zhang, Qinan Wang. 1996. Multiattribute utility function for a comprehensive health status classification system: Health utilities index mark 2. Medical Care 34 702-722.

Von Winterfeldt, Detlof, Ward Edwards. 1986. Decision Analysis and Behavioral Research. Cambridge University Press, Cambridge, U.K.

Weinstein, Milton C., William B. Stason. 1977. Foundations of costeffective analysis for health and medical practices. New England J. Medicine 296 716-721.

Zank, Horst. 2001. Cumulative prospect theory for parametric and multiattribute utilities. Math. Oper. Res. 26 67-81. 